\title{
Partial Discharge Degradation and Breakdown Studies in Polypropylene Films
}

\author{
Benny Thomas and R. S. Nema \\ Department of High Voltage Engineering \\ Indian Institute of Science, Bangalore - 560012 \\ INDIA.
}

\begin{abstract}
Internal partial discharge characteristics are investigated on impregnated polypropylene films containing artificial cavity of well-defined dimensions, immersed in oil. Electrical breakdown studies are carried out at step-wise rising stress to evaluate constants of inverse power law model. Partial discharge pulse distribution patterns are acquired using PC interfaced multichannel analyser and statistical analysis of the discharge pulse spectrum is done by using 3- parameter Weibull distribution function. The results are compared with that for unimpregnated samples in air.
\end{abstract}

\section{INTRODUCTION}

Polypropylene films are extensively used as dielectric in high voltage capacitors. Internal partial discharges cause long term degradation of polymeric insulation. Eventhough, an in-depth study on partial discharges started as early as 1920's, the basic problem of measurement, evaluation, localisation and coherence between partial discharge quantities and the residual life-time remain unsolved. All investigations show that statistical interpretations of partial discharge data is to be considered, since on the one hand, the partial discharge processes are stochastic in nature and, on the other hand, a large amount of data is involved. Statistical methods can be employed, only if equipments capable of collecting and handling the information faster and more efficiently, are available. One of the advantages of multi-channel analyser based computer-aided measuring system is it's ability to process and transform the information into an understandable output. An attempt is made to study the partial discharge degradation and breakdown characteristics in polypropylene-film samples, having a single artificial cavity of known dimensions.

\section{EXPERIMENTAL PROCEDURE}

Electrical grade biaxially oriented polypropylene films of thickness $20 \mu \mathrm{m}$ are cut as circular pieces of $110 \mathrm{~mm}$ diameter. Cylindrical hole of $0.9 \mathrm{~mm}$ diameter is drilled at the centre, using a micro-processor controlled, high speed drilling machine. The electrodes used are $\frac{2 \pi}{3}$ Rogowski profile uniform field brass electrodes of $58 \mathrm{~mm}$ overall diameter and 19 mm radius of curvature, embedded in epoxy (to form $75 \mathrm{~mm}$ overall diameter and $20 \mathrm{~mm}$ overall thickness) except for the flat portion of the electrode surface (exposed effective diameter of the electrode being 48 $\mathrm{mm}$ ). Six drilled films are kept in between two single plane films to form a dielectric bounded artificial cavity of $0.9 \mathrm{~mm}$ diameter and $120 \mu \mathrm{m}$ depth. The test sample is placed in between the grounded bottom electrode and the upper high voltage electrode. A thin film of impregnating oil is manually pasted on each layer to form the impregnated sample and, the electrode-sample assembly is completely immersed in oil. Step-wise rising stress is used to obtain the breakdown characteristics of the sample. The voltage is first increased from zero to 1.1 times the inception voltage and, is maintained at this level for a time interval $t_{s}$ minutes. The voltage is then raised in steps of $500(\mathrm{rms})$ volts and maintained for $t_{s}$ minutes at each step stress, till the sample breakdowns. Time to failure and the applied voltage level at the instant of breakdown are recorded. The time interval $\left(t_{s}\right)$ is chosen to be 1 minute, 3 minutes, 5 minutes and 10 minutes.

Computer aided measurement system is used to monitor the partial discharge pulse distribution patterns. The sample is coupled to a pre-amplifier 
(a narrow band tuned amplifier with mid-band frequency $500 \mathrm{kHz}$ and band width $+/-5 \mathrm{kHz}$ ) through a band-pass filter, a $1000 \mathrm{pF}(100 \mathrm{kV})$ discharge - free coupling capacitor and a tuned detection impedance. The output of the pre-amplifier is amplified using a linear-pulse amplifier (gain : $0.1-1.0$ and time constant : $1-5 \mu s$ and, is fed to an IBM PC interfaced multichannel analyser (EG \& G ORTEC, $U S A)$, where the partial discharge pulses are graded into various channels according to their height, digitized by the analogue to digital converter and, then transmitted to the personal computer (PC). To acquire the pulse distribution pattern, the same stepwise rising stress is applied across the sample, but the final step -voltage level is limited to $2500 \mathrm{~V}$, in view of safety of measuring instruments. Two to five pulse distribution patterns with data acquisition period of 20 seconds each, are recorded in computer floppy diskettes at step voltages of $1.1 \mathrm{Vi}, 1000 \mathrm{~V}$, $1500 \mathrm{~V}, 2000 \mathrm{~V}$ and $2500 \mathrm{~V}$, for different step-time intervals of $1,3,5$ and 10 minutes. Each spectrum contains the data of number of discharges pertaining to 512 different apparent-charge magnitudes. Since the data processing is complicated and, a large capacity of computer memory is needed for on-line analysis, processing of data is done off-line by using another personal computer.

\section{RESULTS AND DISCUSSIONS}

The breakdown stress as well as time to failure for unimpregnated samples in air is decreased by about 50 percent in presence of the cavity (Figure 1 ), whereas the voltage endurance coefficient $(n)$ is reduced from 10.51 to 7.1 (Table 1). For impregnated sample immersed in oil, the breakdown strength and time to failure is decreased by about 68 percent and the $n$-value is reduced from 12.57 to 9.07 . The results show that the internal partial discharges degrade both the unimpregnated and impregnated polypropylene films, for the later, damage is more predominant. The impregnation reduces the amount of long term degradation as indicated by the $n$ value, by an order of 2 for both polypropylene samples with and without cavity, and increases the breakdown strength by 44 percent in the absence of cavity, while by only 10 percent in the presence of cavity. Partial Discharge induced physical and chemical degradation processes on insulating materials are well documented in the literature.

Starr and Endicott [1] proposed the applicability of inverse- power law (IPL) model for linearly increasing stress. Shobha and Nema [2] in their work on internal partial discharge aging on polypropylene films suggested that step-wise rising stress can be used to estimate the constants of IPL model. The step-wise stress considerably reduces the time to failure [3]. The constant $(\mathrm{K})$ of the IPL model is tabulated in Table 1, which is in the same order of magnitude. Since the internal partial discharge is the major aging mechanism in the present experiments and, the $K$ - value indicates a measure of damage to the insulation, it implies that the cumulative damage is independent of the step interval time $\left(t_{s}\right)$. Hence, step-wise rising stress method can be used to estimate the $n$ - value of IPL model. Figures 2 - 4 depict variation of average discharge magnitude $\left(Q_{a}\right)$, number of pulses per second $(\mathrm{N})$ and total discharge magnitude $\left(Q_{t}\right)$ with overvoltage, for step- time intervals of $1,3,5$ and 10 minutes, for both the unimpregnated samples in air and unimpregnated samples in oil, submitted to internal partial discharges. For unimpregnated samples in air, average discharge magnitude steeply reduces with overvoltage and finally, reaches a stabilized value as shown in Figure 2 , whereas number of pulses per second sharply increases and then, reaches a stabilised value (Figure 3). The total discharge magnitude also reaches a stabilised value (Figure 4), independent of the step-time interval. In Figures $2 \& 3$, it can be seen that the average discharge magnitude is highest and, the pulse repetition rate is lowest at inception voltage level, which implies that discharges of larger magnitudes predominate at lower voltage and subsequently, number of lower magnitude discharges increase. Increase in the number of lower magnitude discharges is an indication of aging of the insulation.

For impregnated samples immersed in oil, the pulse rate, average discharge magnitude and total discharge magnitude are much lower, in comparision with the corresponding values for unimpregnated samples in air and, these discharge quantities substantially remain within a small range of magnitude over the entire range of applied voltage levels. It is to be noted that the magnitude of these discharge quantities is comparable with the stabilised values 
of respective quantities for unimpregnated samples in air. Impregnation slows down the detrimental aging processes at the begining, but later, aging becomes comparable with or more than that in unimpregnated samples. It may be due to structural or chemical changes in the polymer, promoted by the impregnating liquid.

The Weibull cumulative probability distribution is frequently used to fit the electrical breakdown data. The 3-parameter Weibull distribution is given by

$$
\ln \ln \left[\frac{1}{1-F(q)}\right]=\beta \ln (q-\gamma)-\beta \ln \alpha
$$

where $\alpha$ is the scale parameter, which represents the discharge magnitude below which lie 63.2 percent of discharge magnitudes, $\beta$ is the shape parameter which is a measure of the dispersen of discharge magnitudes, $\gamma$ is the location parameter which indicates the discharge magnitude below which no discharges occur and $q$ (the discharge magnitude) is the random variable. The pulse distribution data fits 3 -parameter Weibull distribution. The variation of statistical parameters $\alpha \& \beta$ with overvoltage are depicted in Figure 5 for unimpregnated sample in air and, in Figure 6 for impregnated sample in oil. The $\alpha$-values are initially maximum and then, decreases rapidly to a stabilized value, for unimpregnated samples in air. The step-interval time does not affect $\alpha$ except for inception voltage or unaged samples (Figure 5). Figure 6 also shows a similar trend for impregnated sample immersed in oil, where $\alpha$ - values having wide variation with step-time interval at inception voltage level tend to converge and finally, stabilize to a representative value for aged sample, as applied voltage level increases. On the one hand, the scatter of $\alpha$-values with step-time interval and, on the otherhand, a stabilized value with increase in applied voltage level seems to be a good measure to assess the status of an aged sample, for impregnated samples as well as unimpregnated samples

The $\beta$-values are independent of the step-interval time, $t_{s}$. Since the $\beta$ - value is invariable with applied voltage level and step - interval time, the conclusion drawn is that a single breakdown mechanism only is present in the sample. Three parameter Weibull distribution can be used to identify the void discharges which occur in solid insulating materials.

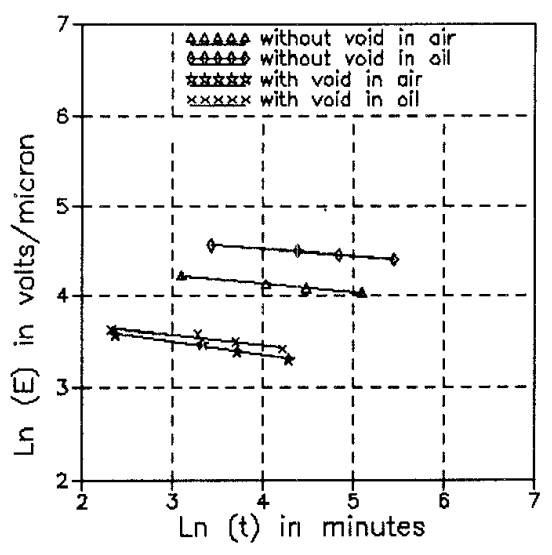

Figure 1. E-t Characteristics

\section{CONCLUSION}

Internal partial discharges considerably reduce the life of impregnated and unimpregnated polypropylene films. Step-wise rising stress method can be used to evaluate constants of inverse power law model. The average discharge magnitude $\left(Q_{a}\right)$, total discharge magnitude $\left(Q_{t}\right)$ and pulse repetition rate $(\mathrm{N})$ stabilize to representative value which indicate the status of aged sample. Number of larger magnitude discharges decrease whereas number of smaller magnitude discharges increase with aging. Impregnation slows down the aging processes. Weibull parameters $\alpha$ and $\beta$ can be used to identify the internal partial discharges and to monitor the status of aging.

\section{REFERENCES}

1. Starr,W.T and Endicott,H.S. "Progressive Stress A New Accelerated Approach to Endurance". AIEE Trans PAS. Vol. 80, 1961, p. 575.

2. Shobha,R and Nema,R.S. "Internal Partial Discharge and Breakdown Characteristics of Thin Polypropylene Films". IEEE CEIDP, 1993, p.408.

3. Laghari,J.R Cygan,P and Khechen,W. "A Short Method of Estimating Life-time of Polypropylene Film using Step- stress Tests". IEEE TransEI. Vol $23(6), 1990, p .1180$.

4. Benny Thomas and Nema,R.S. "Internal Partial Discharge Characteristics in Thin Polypropylene Films". Conference on High Voltage Engineering, IISc, Bangalore, India, 1995,pp.209-212. 
Table 1

E - t Characteristics data

\begin{tabular}{|c|c|c|c|c|c|}
\hline Sample & $\begin{array}{c}n \\
(\#)\end{array}$ & $\begin{array}{c}t_{s} \\
(\min .)\end{array}$ & $\begin{array}{l}\text { Time to } \\
\text { Failure } \\
t(\text { min. })\end{array}$ & $\begin{array}{c}\text { Field } \\
\text { Strength } \\
E\left(V \mu^{-1}\right)\end{array}$ & $\begin{array}{c}\text { Constant } \\
K(\#)\end{array}$ \\
\hline \multirow{4}{*}{ A1 } & \multirow{4}{*}{10.51} & 1 & 21.977 & 68.03 & $4.015 \times 10^{20}$ \\
\hline & & 3 & 56.260 & 61.56 & $3.595 \times 10^{20}$ \\
\hline & & 5 & 87.357 & 59.15 & $3.666 \times 10^{20}$ \\
\hline & & 10 & 164.022 & 56.26 & $4.069 \times 10^{20}$ \\
\hline \multirow{4}{*}{ A2 } & \multirow{4}{*}{7.10} & $\overline{1}$ & 11.516 & 35.52 & $1.173 \times 10^{12}$ \\
\hline & & 3 & 23.424 & 32.14 & $1.173 \times 10^{12}$ \\
\hline & & 5 & 41.338 & 29.67 & $1.173 \times 10^{12}$ \\
\hline & & 10 & 78.320 & 27.11 & $1.173 \times 10^{12}$ \\
\hline \multirow{4}{*}{ B1 } & \multirow{4}{*}{12.57} & 1 & 30.630 & 95.83 & $2.475 \times 10^{26}$ \\
\hline & & 3 & 79.870 & 89.58 & $2.765 \times 10^{26}$ \\
\hline & & 5 & 126.16 & 85.42 & $2.402 \times 10^{26}$ \\
\hline & & 10 & 232.75 & 81.25 & $2.362 \times 10^{26}$ \\
\hline \multirow{4}{*}{ B2 } & \multirow{4}{*}{9.07} & 1 & 12.071 & 37.34 & $2.193 \times 10^{15}$ \\
\hline & & 3 & 18.997 & 35.52 & $2.193 \times 10^{15}$ \\
\hline & & 5 & 39.244 & 32.79 & $2.193 \times 10^{15}$ \\
\hline & & 10 & 81.068 & 30.27 & $2.193 \times 10^{15}$ \\
\hline
\end{tabular}

$n$ : Endurance Coefficient

$t_{s}:$ Step-time Interval

A1 : Unimpregnated Sample in Air Without Void

A2: Unimpregnated Sample in Air With Void

B1 : Impregnated Sample in Oil Without Void

B2 : Impregnated Sample in Oil With Void

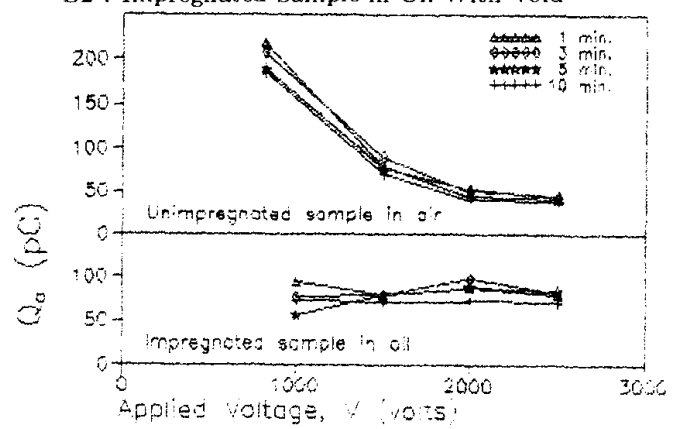

Figure 2. Variation of average discharge magnitude with applied voltage

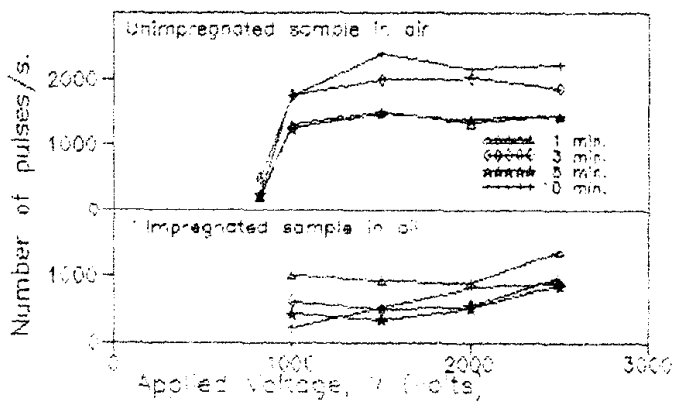

Figure 3. Variation of pulse repetition rate with applied voltage

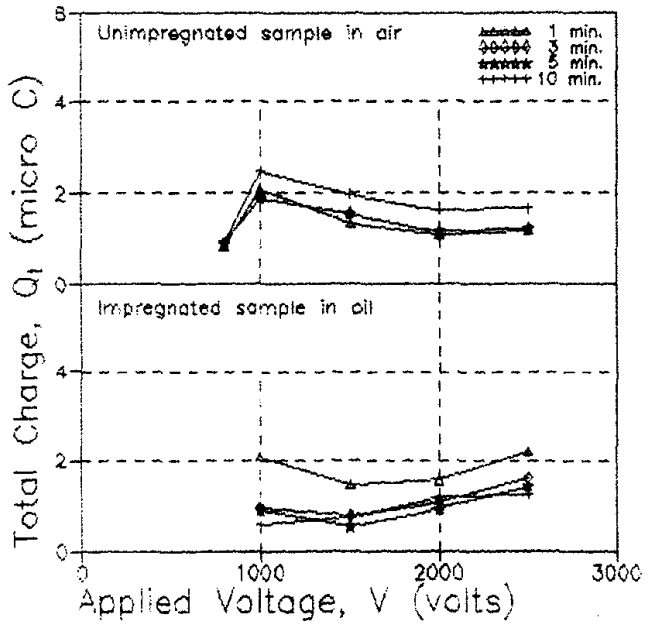

Figure 4. Variation of total discharge magnitude with applied voltage

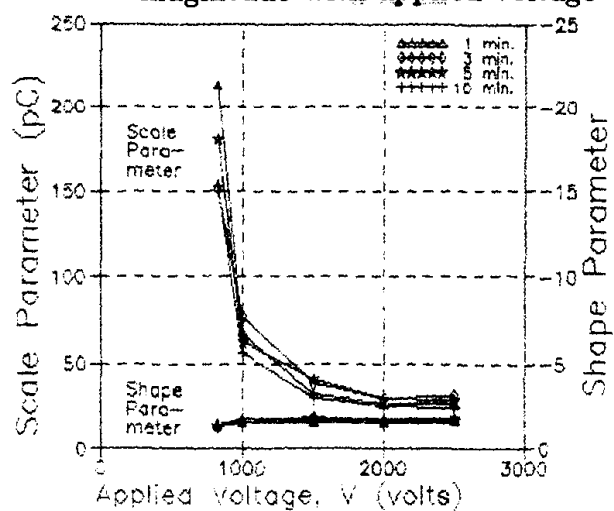

Figure 5. Variation of scale parameter with applied voltage for umimpregnated sample in air

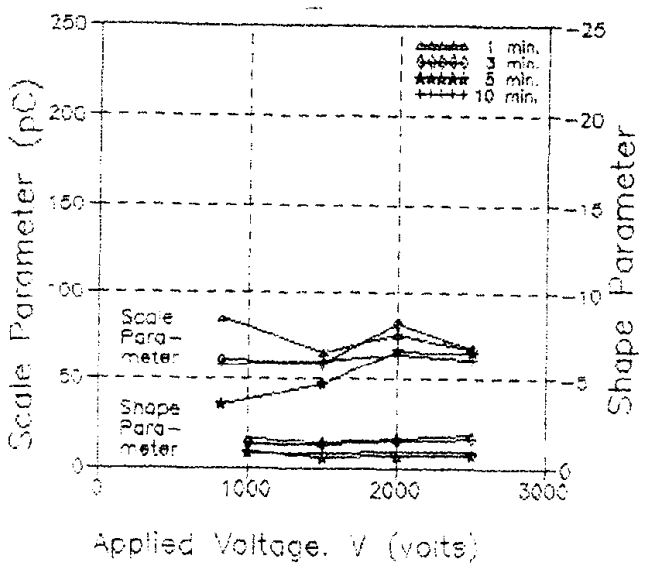

Figure 6. Variation of total discharge magnitude with applied voltage 